Case Report

\title{
A Case of Bacteremia and Meningitis Associated with Piperacillin-Tazobactam Nonsusceptible, Ceftriaxone Susceptible Escherichia coli during Strongyloides Hyperinfection in an Immunocompromised Host
}

\author{
Santosh Dahal, Jeffrey Lederman, Jesse Berman, Marius Viseroi, and Stephen Jesmajian \\ Department of Internal Medicine, Albert Einstein College of Medicine, Montefiore Medical Center, New Rochelle, NY 10801, USA \\ Correspondence should be addressed to Santosh Dahal; sdahal@montefiore.org
}

Received 15 June 2017; Revised 14 September 2017; Accepted 17 October 2017; Published 22 November 2017

Academic Editor: Alexandre R. Marra

Copyright (C) 2017 Santosh Dahal et al. This is an open access article distributed under the Creative Commons Attribution License, which permits unrestricted use, distribution, and reproduction in any medium, provided the original work is properly cited.

\begin{abstract}
Strongyloidiasis is an emerging parasitic infection with intriguing epidemiology, presentation, and clinical management. We report a case of hyperinfection syndrome complicated by E. coli bacteremia and meningitis with one of the isolates showing a unique resistance pattern recently being recognized. This report describes the aspect of invasive bacterial infections in strongyloidiasis and highlights the unique susceptibility pattern of the E. coli isolate and the extreme caution required during the antibiotic therapy.
\end{abstract}

\section{Introduction}

Invasive bacterial infections, including meningitis by enteric and other commensal bacteria, are a known complication of severe Strongyloides spp. infection. Mortality rates as high as $60 \%$ and $68.5 \%$ have been reported for hyperinfection syndrome and disseminated infection, respectively [1]. Furthermore, Gram-negative bacillary meningitis by itself is a serious infection with a case fatality rate between 15 and 40\% [2]. Eradication of the parasite is pivotal in the management, and it can be challenging in an immunocompromised host. Appropriate antibiotic therapy is the cornerstone in the management.

\section{Case Presentation}

The case is a 59-year-old Hispanic female born in Mexico with a medical history of insulin-dependent type 2 diabetes and rheumatoid arthritis on methotrexate, abatacept, and prednisone. Her prednisone dose was increased because of corneal transplant two days prior to admission. The patient was seen in the emergency department multiple times over the preceding month for abdominal pain, diarrhea, and vomiting and was treated with ciprofloxacin and metronidazole a week prior. She presented with lethargy, temperature $102^{\circ} \mathrm{F}$, heart rate $121 \mathrm{bpm}$, and blood pressure 102/80 $\mathrm{mmHg}$. Her Glasgow Coma Scale (GCS) score was 15/15, and meningeal signs were absent. The blood leukocyte count was 16,600 cells/mcL, serum sodium was $121 \mathrm{mmol} / \mathrm{L}$, potassium was $3.4 \mathrm{mmol} / \mathrm{L}$, chloride was $89 \mathrm{mmol} / \mathrm{L}, \mathrm{CO}_{2}$ was $10 \mathrm{mmol} / \mathrm{L}$, and blood sugar was $222 \mathrm{mg} / \mathrm{dl}$. Arterial blood gas analysis showed triple acid-base disturbance with high anion gap metabolic acidosis, metabolic alkalosis, and respiratory alkalosis $\left(\mathrm{pH} 7.35, \mathrm{pCO}_{2}\right.$ 13 , and $\mathrm{HCO}_{3}$ 7.1). Urine showed large ketones. CT scan of the abdomen showed possible colitis with thickening of the colon. She was started on insulin protocol after supplementing potassium. She was started on vancomycin $1.25 \mathrm{~g}$ every 12 hours (q12 hr) and piperacillin-tazobactam (PTZ) $3.37 \mathrm{~g}$ every 6 hours (q6 hr). Immunosuppressive agents were put on hold. She became more acidotic and tachypneic requiring intubation to prevent respiratory fatigue. On day 2 (D2), blood cultures grew Gram-negative bacilli (GNB). Suspicion of strongyloidiasis was high in the setting of Gram-negative bacteremia and a recent history of persistent GI symptoms in a patient from an endemic region. Single dose of ivermectin 200 micrograms/kg was given empirically. On D3, stool and 
TABLe 1: Antibiotic sensitivity reports for E. coli isolates (MicroScan WalkAway, NBC44).

\begin{tabular}{lcc}
\hline Test panel & $\begin{array}{c}\text { E. coli (blood culture) MIC/KB value (specimen } \\
\text { collected on } 12 / 07 / 2016)\end{array}$ & $\begin{array}{c}\text { E. coli (CSF culture) MIC/KB value (specimen } \\
\text { collected on 12/14/2016) }\end{array}$ \\
\hline Ampicillin/sulbactam & Resistant $(R)>16 / 8$ & $R>16 / 8$ \\
Amikacin & Sensitive $(S) \leq 16$ & $S \leq 16$ \\
Ampicillin & $R>16$ & $R>16$ \\
Aztreonam & $S \leq 8$ & $S \leq 8$ \\
Ceftriaxone & $S \leq 8$ & $S \leq 8$ \\
Cefoxitin & $S \leq 8$ & $S \leq 8$ \\
Cefazolin & $S \leq 8$ & $R>16$ \\
Ciprofloxacin & $R>2$ & $R>2$ \\
Ertapenem & $S \leq 1$ & $S \leq 1$ \\
Gentamicin & $S \leq 4$ & $S \leq 4$ \\
Imipenem & Not tested & $S \leq 4$ \\
Piperacillin/tazobactam & $S \leq 16$ & $R>64$ \\
Trimethoprim/sulfamethoxazole & $R>2 / 38$ & $R>2 / 38$ \\
\hline
\end{tabular}

sputum microscopy reported larvae of Strongyloides spp. GNB from blood culture was identified as E. coli, which was sensitive to cefazolin and piperacillin-tazobactam (PTZ) (Table 1). Hence, PTZ was continued.

She improved clinically, remained afebrile, and got extubated on D4, was started on oral diet on D5, and was moved out of ICU to regular floor on D6. Serology for Strongyloides was reported to be positive for immunoglobulin (Ig) A. On day 7, PTZ was switched with cephalexin $500 \mathrm{mg}$ q6 hr. Early the next morning, she was found to have rapidly worsening mental status and was reintubated. Cephalexin was switched with cefepime $2 \mathrm{~g}$ q8 hr and ampicillin $2 \mathrm{~g} \mathrm{q} 4 \mathrm{hr}$. Lumbar puncture (LP) was done, and CSF showed GNB, WBC 330 cells/mcL with $98 \%$ granulocytes and $2 \%$ monocytes, RBC 250 cells/mcL, glucose less than $10 \mathrm{mg} / \mathrm{dl}$, and protein $458 \mathrm{mg} / \mathrm{dl}$. Ampicillin was stopped. Repeat stool microscopy remained positive for Strongyloides spp. Ivermectin dose was increased to $400 \mathrm{mcg} / \mathrm{kg}$ daily in $40 \%$ ethanol to increase absorption, and albendazole $400 \mathrm{mg}$ q12 hr was also added. On D10, CSF culture reported E. coli resistant to PTZ and cefazolin but sensitive to ceftriaxone (CTX) (Table 1). Identification and sensitivity were done by using the MicroScan WalkAway 40 SI system with Negative combo 44 panels. Cefepime was continued. Her mental status did not improve, and the electroencephalogram was reported to be consistent with vegetative state with cerebral dysfunction. Repeat LP after 2 weeks showed WBC 82 cells/mcL with granulocytes $53 \%$, lymphocytes, $46 \%$, and monocytes $1 \%$, RBC 9 cells $/ \mathrm{mcL}$, glucose $51 \mathrm{mg} / \mathrm{dl}$, total protein $81 \mathrm{mg} / \mathrm{dl}$, and $\mathrm{LDH} 192$, and Gram staining and culture were negative. Repeated stool exams were negative for larvae. The patient received 14 days of ivermectin and 7 days of albendazole. She did not improve and underwent palliative extubation on D19 and died 10 days later.

\section{Discussion}

Strongyloidiasis is an emerging parasitic infection in developing countries of tropical and subtropical regions with small endemic areas in the temperate region. However, according to recent epidemiological studies, there is some endemicity even in developed countries, including the United States [3]. Epidemiology is complicated by international travel and migration patterns, and global data are considered inadequate [4]. In our case, the patient was born and grew up in Mexico and had a history of multiple travels to Mexico.

The life cycle of this helminthic parasite is unique and complex, with filariform larvae penetrating the skin and ending up in the gut from various routes involving connective tissue, bloodstream, and lungs. Their ability to cause autoinfection is characteristic, which is also the mechanism for hyperinfection. The clinical course is commonly chronic and symptomatically mild, but in immunosuppressed hosts, hyperinfection syndrome characterized by severe disease with high parasitic load and disseminated infection involving internal organs can occur which could be fatal [5]. Diagnosis is difficult and usually late because of variable presentation. In nonendemic settings, a very high index of suspicion is required for diagnosis. In this case, chronic GI symptoms were suggestive, and she had strong risk factors such as the use of abatacept and prednisone and diabetes [6]. No evidence could be found as to whether she was screened for Strongyloides spp. before she was started on immunosuppressive therapy, but the screening could have been lifesaving. History of persistent GI symptoms and Gramnegative bacteremia without other sources of infection aroused high suspicion of strongyloidiasis, so we treated her with one dose of ivermectin while waiting for stool microscopy. Our decision to start treatment prior to obtaining diagnostic evidence was based on clinical judgement, and there is no evidence regarding empirical treatment of strongyloidiasis.

Invasive bacterial infections are a well-known complication of strongyloidiasis, which is explained by bacterial translocation on the nematode's surface as it penetrates the intestinal mucosa [7]. According to one study, out of 30 patients with $S$. stercoralis, 16 had invasive infections, including sepsis, meningitis, pneumonia, peritonitis, and 
endocarditis caused by enteric bacteria and Candida spp. [8]. Although pathogenesis is still unclear, high-grade bacteremia is key to meningitis [2]. Bacterial isolates from meningitis associated with strongyloidiasis are mostly the intestinal commensals including Escherichia coli, Streptococcus bovis, Klebsiella pneumoniae, Lactococcus lactis, Streptococcus sanguinis, Enterococcus faecium, and Staphylococcus warneri $[9-11]$. We used broad-spectrum therapy with PTZ and vancomycin for our patient until blood culture reported growth of GNB.

A week after hospitalization, when the patient was moved out of ICU to regular floors and a day after switching from PTZ to cephalexin, the patient had rapid deterioration of mental status and was found to have meningitis from E. coli resistant to PTZ and cefazolin but susceptible to CTX. This situation could have resulted from partially treated CNS infection because of ineffective CNS concentration of PTZ, leading to selection of resistant strains or failure to control high inoculum of E. coli infection with seeding of the central nervous system. Clinical worsening while the patient was switched from PTZ to cephalexin which was active for less than 24 hours was most likely coincidental than contributory to worsening of the disease. It has been reported that the clinical use of $\beta$-lactam $/ \beta$-lactamase inhibitor combinations results in the selection of point mutants in TEM penicillinases resistant to inhibitors, referred to as inhibitor-resistant TEMs (IRTs) which are generally susceptible to cephalosporins. Strains with complex mutant TEMs (CMTs) that combine ESBL and IRT mutations are resistant to both groups of antibiotics [12]. In this case, E. coli isolated from CSF was not an ESBL producer, was resistant to cefazolin, and was sensitive to CTX. A study has reported $4.1 \%$ of E. coli and $4.4 \%$ of $K$. pneumoniae isolates from bloodstream infections with resistance to PTZ and susceptibility to CTX. Most of these patients were hospital inpatients at infection onset and had recently received antibacterial agents, including $\beta$-lactam/ $\beta$-lactamase inhibitor (BL/BLI), and gastrointestinal translocation was the most common presumed source. They have been found to be nonclonal and associated with deleted or dysfunctional porins contributing to the observed resistance pattern [13, 14].

The patient was initially started on PTZ to cover for Gram-negative infection including Pseudomonas spp. In a patient presenting with diabetic ketoacidosis with potential abdominal source of infection and without any meningeal signs, concern for meningitis was not significant. PTZ was switched with cephalexin as per the sensitivity report once the patient clinically improved and completed 7 days of intravenous antibiotics. Since there was no suspicion of meningitis, switching to oral therapy seemed appropriate. The optimal duration of antibiotic therapy for bloodstream infections is unknown, and shorter duration of therapy has been demonstrated to be equally effective [15]. With the view of antibiotic stewardship, there was a missed opportunity to de-escalate from PTZ to ceftriaxone once blood culture was reported. Retrospectively switching to ceftriaxone and increasing dose to the meningitic level could have altered the course of disease. When mental status worsened and meningitis was suspected, the patient was started on cefepime and ampicillin. Cefepime was continued even after the sensitivity results because we wanted to suppress the Gramnegative rods in the gut.

Meningitis could have also been caused by reinfection during the continued migration of larvae. There is a strong possibility that clinical worsening and the poor outcome were from the failure to control spread of the larvae rather than the effect of bacterial infection alone. Treatment of invasive bacterial infections is tied with parasite cure because failure in doing so poses risk for recurrent infections [11]. Ivermectin is the drug of choice, but there is no consensus regarding the dosing, and cure rates vary between 55 and 100\% [16]. In our case, stool microscopy was still positive for larvae on D7 after 5 days of ivermectin $200 \mathrm{mcg} / \mathrm{kg}$ daily dose after which dosing was increased to $400 \mathrm{mcg} / \mathrm{kg}$ daily on $40 \%$ ethanol to increase absorption, and albendazole was also added.

Limitation of this report is the lack of further testing and characterization of the two E. coli isolates which would have provided answers as to how closely they were related and the possible mechanism of the resistance pattern. It is important for the clinical practitioners to be aware of the occurrence of such a resistance pattern and the prevalence in their practice settings. Enteric bacteria and the bowel flora can cause invasive bacterial infections including meningitis during strongyloidiasis, and there should be high suspicion of meningitis in these cases. Antibiotic therapy should be decided taking into account high rates of resistance found among enteric bacteria and the risk of reinfection until the parasitic infection is cured.

\section{Conflicts of Interest}

The authors declare that they have no conflicts of interest.

\section{References}

[1] D. Buonfrate, A. Requena-Mendez, A. Angheben et al., "Severe strongyloidiasis: a systematic review of case reports," BMC Infectious Diseases, vol. 13, no. 1, p. 78, 2013.

[2] K. S. Kim, "Human meningitis-associated Escherichia coli," EcoSal Plus, vol. 7, no. 1, 2016.

[3] L. A. Marcos, A. Terashima, H. L. Dupont, and E. Gotuzzo, "Strongyloides hyperinfection syndrome: an emerging global infectious disease," Transactions of the Royal Society of Tropical Medicine and Hygiene, vol. 102, no. 4, pp. 314-318, 2008.

[4] World Health Organization, Strongyloidiasis, WHO, Geneva, Switzerland, 2017.

[5] L. A. Marcos, A. Terashima, M. Canales, and E. Gotuzzo, "Update on strongyloidiasis in the immunocompromised host," Current Infectious Disease Reports, vol. 13, no. 1, pp. 35-46, 2011.

[6] S. Lim, K. Katz, S. Krajden, M. Fuksa, J. S. Keystone, and K. C. Kain, "Complicated and fatal Strongyloides infection in Canadians: risk factors, diagnosis and management," Canadian Medical Association Journal, vol. 171, no. 5, pp. 479-484, 2004.

[7] W. G. Simpson, D. C. Gerhardstein, and J. R. Thompson, "Disseminated Strongyloides stercoralis infection," Southern Medical Journal, vol. 86, no. 7, pp. 821-825, 1993. 
[8] M. N. Al-Hasan, M. McCormick, and J. A. Ribes, "Invasive enteric infections in hospitalized patients with underlying strongyloidiasis," American Journal of Clinical Pathology, vol. 128, no. 4, pp. 622-627, 2007.

[9] T. Shimasaki, H. Chung, and S. Shiiki, "Five cases of recurrent meningitis associated with chronic strongyloidiasis," American Journal of Tropical Medicine and Hygiene, vol. 92, no. 3, pp. 601-604, 2015.

[10] L. Zammarchi, F. Montagnani, G. Tordini et al., "Persistent strongyloidiasis complicated by recurrent meningitis in an HTLV seropositive Peruvian migrant resettled in Italy," American Journal of Tropical Medicine and Hygiene, vol. 92, no. 6, pp. 1257-1260, 2015.

[11] R. N. Incani, M. Hernández, J. Cortez, M. E. E. González, and Y. D. Salazar, "Staphylococcus warneri meningitis in a patient with Strongyloides stercoralis hyperinfection and lymphoma: first report of a case," Revista do Instituto de Medicina Tropical de Sao Paulo, vol. 52, no. 3, pp. 169-170, 2010.

[12] J. Lee, C. E. Oh, E. H. Choi, and H. J. Lee, "The impact of the increased use of piperacillin/tazobactam on the selection of antibiotic resistance among invasive Escherichia coli and Klebsiella pneumoniae isolates," International Journal of Infectious Diseases, vol. 17, no. 8, pp. e638-e643, 2013.

[13] T. Baker, D. P. Calfee, L. Westblade, S. Jenkins, B. N. Kreiswirth, and M. Satlin, "Characterization of piperacillin-tazobactamnon-susceptible, but ceftriaxone-susceptible, Escherichia coli and Klebsiella pneumoniae bloodstream infections," Open Forum Infectious Diseases, vol. 3, no. 1, p. 2014, 2016.

[14] J. R. Mediavilla, Z. Schneider, C. Nwaigwe et al., "Molecular characterization of piperacillin-tazobactam (TZP)-resistant Escherichia coli susceptible to cephalosporins, monobactams, and carbapenems," Open Forum Infectious Diseases, vol. 2, no. 1, p. 1181, 2015.

[15] T. C. Havey, R. A. Fowler, and N. Daneman, "Duration of antibiotic therapy for bacteremia: a systematic review and meta-analysis," Critical Care, vol. 15, no. 6, p. R267, 2011.

[16] T. Mendes, K. Minori, M. Ueta, D. C. Miguel, and S. M. Allegretti, "Strongyloidiasis current status with emphasis in diagnosis and drug research," Journal of Parasitology Research, vol. 2017, Article ID 5056314, 13 pages, 2017. 


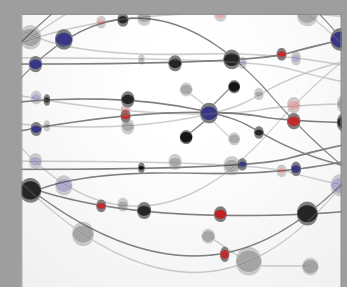

The Scientific World Journal
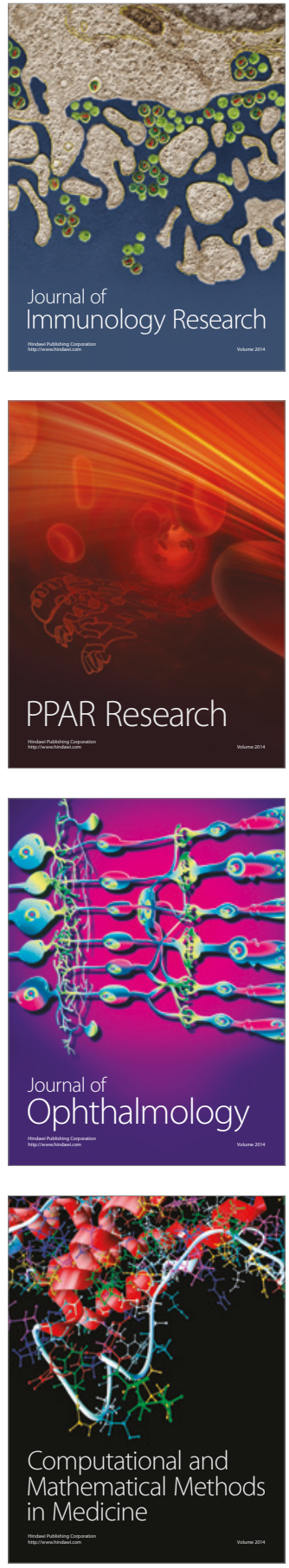

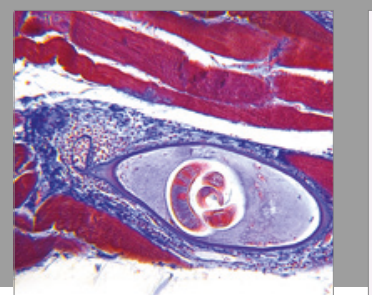

Gastroenterology Research and Practice
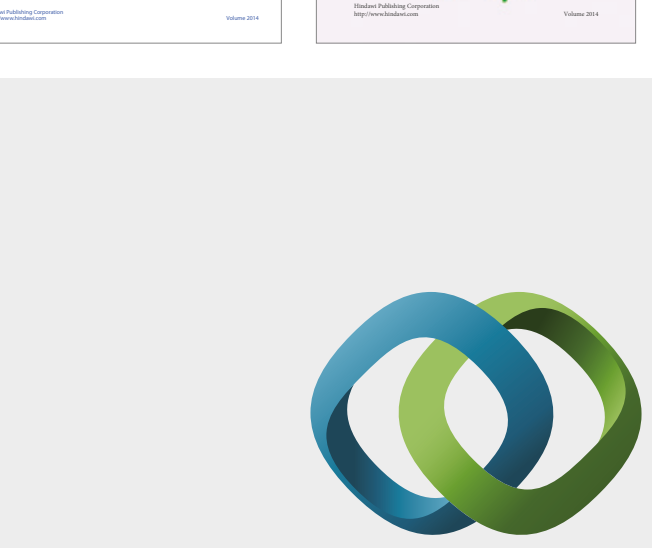

\section{Hindawi}

Submit your manuscripts at

https://www.hindawi.com
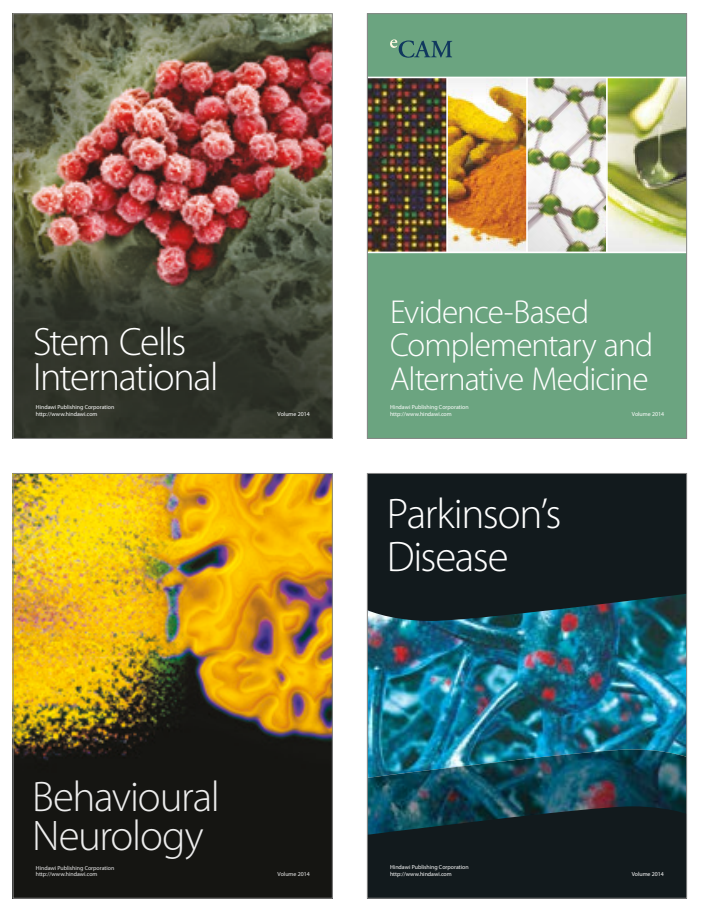
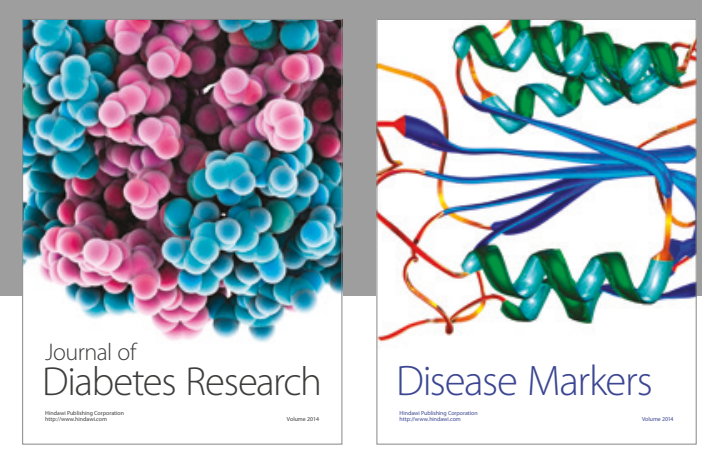

Disease Markers
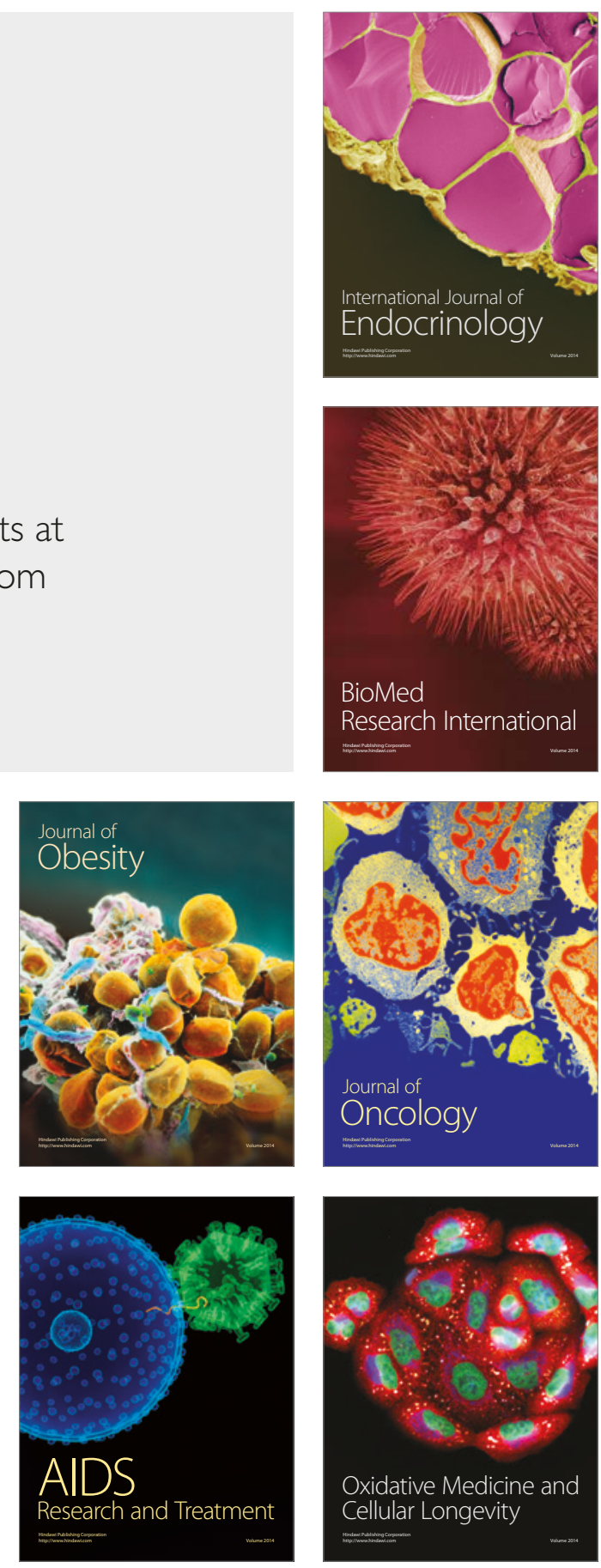